\title{
IgA and IgG Antitransglutaminase 2 Antibodies in the Diagnosis of Celiac Disease
}

\author{
Mónica Villanueva ${ }^{1,2}$, Marianela Rojas ${ }^{1,2}$, Magdalena Araya ${ }^{1, *}$ \\ ${ }^{1}$ Gastroenterology Unit, INTA, University of Chile, Santiago, Chile \\ ${ }^{2}$ Pediatrician, fellow Specialty Program on Gastroenterology and Nutrition, \\ Faculty of Medicine, University of Chile, Santiago, Chile \\ *Corresponding author: maraya@inta.uchile.cl
}

\begin{abstract}
Screening for celiac disease (CD) dramatically improved when techniques able to measure blood autoantibodies against tissue transglutaminase 2 (TTG) were developed. Although typically increased in CD, these antibodies are not pathognomonic since they are also detected in several other autoimmune processes. IgA deficiency among celiac patients is more frequent than in general population (up to $25 \%$ vs $1-3 \%$ ). This led to develop kits able to measure IgG-TTG, which until today represent a helpful diagnostic tool during diagnosis of CD in IgA deficient individuals. Today, commercial kits measuring IgG- TTG (and other) antibodies are widely available, are frequently used and create confusion in diagnosing CD in IgA-sufficient individuals. This is attributed to the fact that sensitivity and specificity of IgG-TTG is lower when applied to IgA-sufficient persons, and also because IgG-TTG is detected in several autoimmune disorders, with variable frequency and isotypes depending on the condition. Evidence analyzed indicate that to date available data: i) is insufficient to understand the difference of classes and subclasses detected in $\mathrm{CD}$ and other autoimmune conditions; ii) does not support the use of IgG-TTG for diagnosing $\mathrm{CD}$ in IgA-sufficient individuals and therefore iii) $\operatorname{IgG}$ should not be used in the routine diagnostic process of $\mathrm{CD}$.
\end{abstract}

Keywords: celiac disease, transglutaminase 2, autoimmunity, IgA deficiency, IgG-TTG

Cite This Article: Mónica Villanueva, Marianela Rojas, and Magdalena Araya, "IgA and IgG Antitransglutaminase 2 Antibodies in the Diagnosis of Celiac Disease." International Journal of Celiac Disease, vol. 5, no. 2 (2017): 43-47. doi: 10.12691/ijcd-5-2-7.

\section{Introduction}

Celiac disease (CD) is a systemic immune mediated disorder triggered by gluten and prolamines present in wheat, barley and rye in genetically susceptible individuals. Characterized by variable degrees of inflammatory enteropathy, it may present a wide range of digestive and extra digestive symptoms and the presence of specific but not pathognomonic blood autoantibodies [1,2]. Both the extra digestive symptoms and blood autoantibodies are also detected in other autoimmune disorders with variable frequencies (see below). The number of people diagnosed with $C D$ has increased in the last decades due to availability of better diagnostic tools and also to improved awareness among professionals and the general population. There is evidence that suggest that the real frequency of $\mathrm{CD}$ has also increased [3]. The only efficacious treatment of $\mathrm{CD}$ is maintaining a strict, permanent gluten-free diet for life. Surveillance of this diet during follow up is difficult because there is no objective way to measure actual gluten ingestion; this is especially so during the transition period of adolescence [4]. None of the existing techniques are completely reliable, including measurements of blood autoantibodies, interviews, questionnaires or the more recently described measurement of 33-mer peptide in feces/urine [5,6] or alkylresorcinols in urine [7], which await validation. At present and both for diagnosis and gluten-free diet control during follow up, measuring blood autoantibodies mainly against transglutaminase 2 (TTG) is the most recommended test worldwide. The routine technique consists of measuring IgA-TTG, but because IgA deficiency is more frequent among celiac patients than in general population, kits that measure IgG type TTG have also been developed. Since appearance of these latter, determination of blood IgG-TTG rapidly became the best choice for assessing $\mathrm{CD}$ in IgA deficient individuals [8].

Numerous companies currently commercialize kits that measure $\mathrm{IgG}$ versions of $\mathrm{CD}$ related autoantibodies; unfortunately, widespread use of these kits has led to confusion when interpreting results in IgA-sufficient individuals assessed for $\mathrm{CD}$. This led us to review the available data on TTG, types and isotypes of antibodies clinically used, the relation between TTG and autoimmunity and between $\operatorname{Ig} \mathrm{A}$ deficiency and $\mathrm{CD}$, to finally conclude on the usefulness of measuring IgG-TTG during the diagnostic process of $\mathrm{CD}$. As the information available is scant and does not allow analysis or comparison between the different types and isotypes of the different antibodies (including anti gliadin, anti endomysial, anti deamidated gliadin peptides), this review includes only IgA and $\operatorname{IgG}$ antibodies against TTG. The 
web search used the words related to "digestive" and "celiac disease".

Literature search combined the terms IgA- and IgG- "antitransglutaminase (TTG), antigliadin (AGA), antiendomysial (EMA), deamidated gliadin peptides" (DGP) with specific terms, including "celiac disease", "autoimmunity" and specific autoimmune disorders using accessible databases including PubMed, Medline, Cochrane Library and BioSciences Information.

\subsection{Transglutaminases}

These are a family of enzymes related by structure and function that catalyze posttranslational modifications in eukaryotic proteins of plants and mammals [9]. TTG is ubiquitously expressed and has multiple functions supplemental to its protein crosslinking ability [10]. It is recognized as the most important autoantigen detected in blood of celiac patients [11]. However, it may be also increased in processes like fibrosis, atherosclerotic plaques formation, metastatic cells and several autoimmune disorders [9]. Anti-transglutaminase antibodies would be locally induced in the small intestinal mucosa by IgA-producing plasma cells [12]. Epitope-mapping studies identify relevant regions both in the enzyme core $[13,14]$ and amino terminus [14]. TTG has a relevant role in the pathogenesis of $\mathrm{CD}$ deamidating the gliadin peptides that will subsequently bind HLA- restricted groves in HLA-DQ2 and DQ8 (Figure 1). Proinflammatory signals originated from the various mucosal cells will contribute to increase TTG production. IgA-TTG are most studied in $\mathrm{CD}$, but other isotypes can also be found in the condition, like IgG-TTG, which has been described, but insufficiently characterized [8]

\subsection{TTG and ELISA Kits}

Antitransglutaminase antibodies are usually measured by ELISA technique; it was first developed in guinea pigs and later human recombinant protein was used. Both have high sensitivity and specificity, over $90 \%$ in children and adults [11]. Sensitivity and specificity are lower for measurements of IgG-TTG; Comerford et al described IgG-TTG positivity was $22 \%$ among pediatric patients and $47 \%$ in adults [15]. Despite these figures, it is interesting that IgG-TTG values were always higher in patients with untreated $\mathrm{CD}$ and both in children and adults results were higher in patients than in controls.

At present, kits measuring different antibodies (like IgA-TTG plus IgG-TTG or TTG plus deamidated gliadin peptides) have been developed. Those in favor of measuring IgA- and IgG- TTG at the same time argue that this will help diagnosing $\mathrm{CD}$ in all persons, independent of their IgA status [16]. However, studies assessing $\operatorname{IgG}$ are so scarce and sensitivity and specificity yielded by these kits lower than those obtained for IgA-TTG that recommendations and clinical Guidelines currently advice the use of IgA-TTG as the first, cost- effective and more reliable method to detect celiac individuals $[1,2]$.

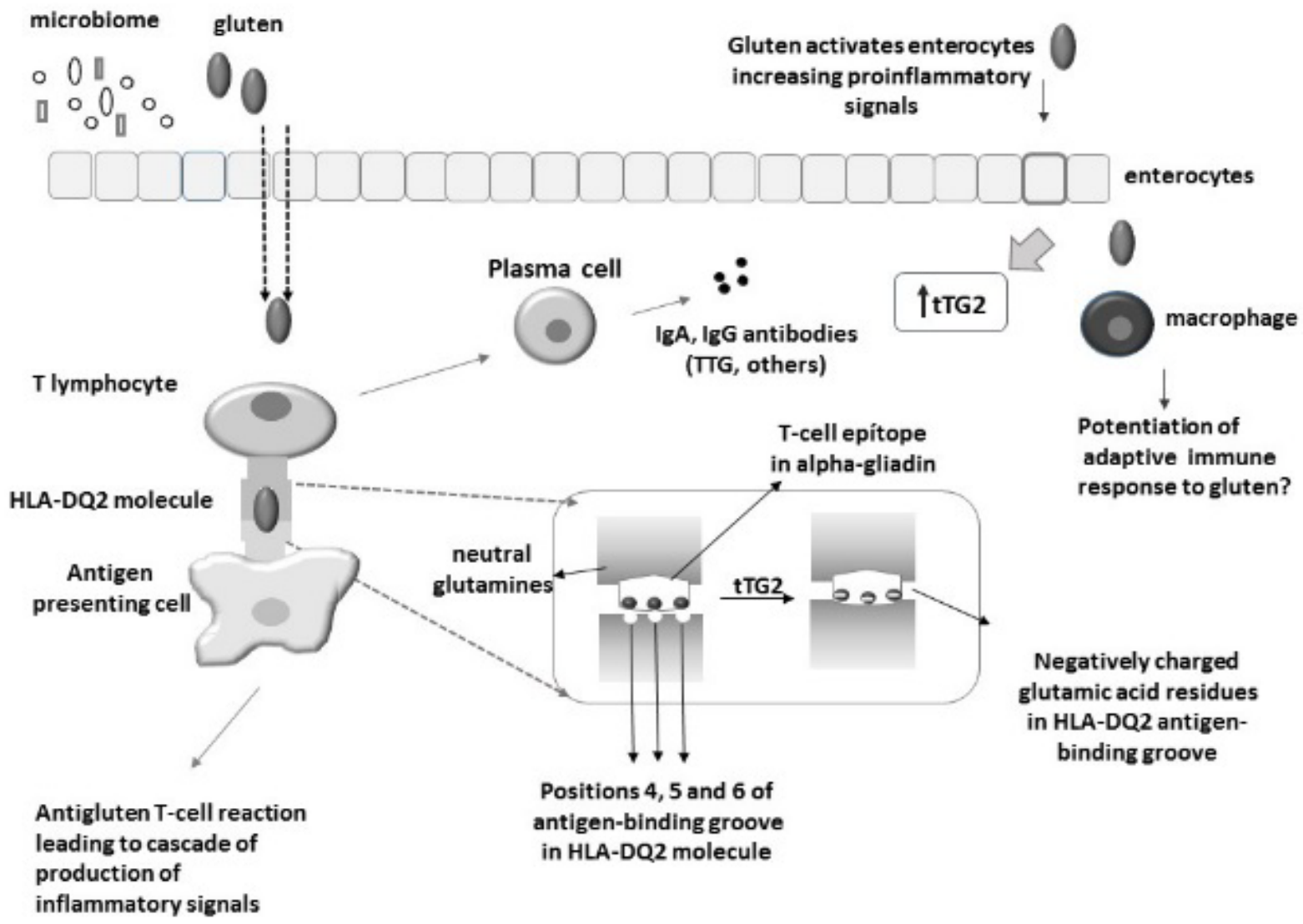

Figure 1. Tissue transglutaminase 2 (TTG2) in the dysregulation of celiac disease. Gluten peptides resist gastrointestinal degradation due to their high proline content. The microbiota may act modifying gluten proteolysis and the net production of immunogenic peptides, which cross the epithelium and reach the lamina propria. TTG2 catalyzes deamidation of these peptides, which results in negatively charged molecules that then are more efficient binding to HLA-DQ2 or -DQ8 molecules on antigen presenting cells. This initiates an anti-gluten T-cell response with release of pro inflammatory signals leading to epithelial damage. Gluten enterocytes and macrophages activation will increase proinflammatory signals that will expand TTG production 
When screening for $\mathrm{CD}$ age is one of the factors influencing the results obtained. When screening for CD age is one of the factors influencing the results obtained. Using IgA-TTG in celiac patients sensitivity and specificity have been described at 98.1\% (95\% CI: 90.1\% $-99.7 \%)$ and $98.0 \%$ (95\% CI: $95.8-99.1)$ in adults [17,18] and 95.7\% (CI: 90.3-98.1) and 99.0\% (CI: 94.6-99.8) in children $[19,20]$, respectively.

\section{IgA Deficiency and Auto-antibodies in CD}

The relation between $\operatorname{IgA}$ deficiency and $\mathrm{CD}$ is currently well established $[21,22]$. In Europe, prevalence of $C D$ is $0.5-6 \%$ in general population and it increases up to $25 \%$ among celiac patients [23]. Estimates are that $2-5 \%$ of celiac individuals are IgA deficient [24]. Therefore, it is certainly important to be able to measure IgG-TTG in patients with insufficient IgA levels who are in the diagnostic process of CD. In IgA deficient celiac patients IgG-TTG determination has yielded sensitivity and specificity that vary between 68 and $100 \%$, respectively, but sensitivity is lower in $\operatorname{IgA}$ sufficient patients [11]. Also in IgA-deficient patients, specificity of $100 \%$ and sensitivity of $90-96 \%$ have also been reported [25], however, among patients exhibiting a wide range of small intestinal lesions the negative predictive value decreased. These limitations have always been and continue being relevant in population studies, which often cannot confirm the diagnosis by biopsy. This evidence has led to agree that IgG-TTG antibodies are not satisfactory to search for $\mathrm{CD}$ in IgA-sufficient individuals $[8,26]$.

\section{Autoimmunity}

One of the problems measuring anti transglutaminase autoantibodies for diagnosing $\mathrm{CD}$ is that although they are typical of this disease they can also be found in non-celiac conditions, mainly autoimmune disorders [27], AIDS [28] and terminal phases of cardiac insufficiency [29]. In non-celiac patients IgG type antibodies are frequent and there is no direct relation between the antibodies type found, either IgA- or IgG-. For example, IgG-TTG in absence of IgA-TTG has been described in patients with DMT1 [30]. Positive IgA-TTG and negative IgA-EMA were reported in patients with Crohn's disease [31], with unclear results on the presence of IgG-TTG [32]. There are reports in rheumatoid arthritis describing patients positive for IgA-TTG and negative for IgA-EMA [33] or IgG-TTG [34]. Also, IgA- and/or IgG- TTG have been reported in systemic lupus erythematosus [32,35], granulomatosis with polyangiitis [36], ankylosing spondylitis (IgA and IgG) and psoriatic arthritis (IgA and IgG) [37]. Differences observed between epitope specificity and the IgA- or IgG- TTG isotype observed may derive from the different mechanisms acting on the enzyme during the process that leads to autoimmunity

\subsection{TTG, IgG-TTG and Antibodies Subclasses}

It must be kept in mind that IgG-TTG described in autoimmune disorders are of different isotypes and these may be of a predominant subclass or a mixture of them. In $\mathrm{CD}$ the predominant subclass is IgG1 [38]. This subclass is also found in DMT1, but it is infrequent in Crohn's disease and in granulomatosis with polyangiitis [15]. Studies analyzing IgG subclasses are quite scarce. Comerford [15] analyzed IgG-TTG by age and showed that IgG-TTG levels were highest in celiac children 0-4 years of age (which coincides with Agardth study [39]) and significantly higher compared to that of $\mathrm{CD}$ patients aged $4-8$ years $(p=0.0225)$, and adults celiacs $(p=0.0009)$ IgG2 was present in $35 \%$ of patients, IgG3 was greater in adults than in children and IgG4 was higher among children. Distribution of IgG subclasses was similar in diabetic patients but different from patients with Crohn's disease, where IgG3 and IgG4 predominated (69\%), while in granulomatosis with polyangiitis IgG1 was always negative and positivity was given by $\mathrm{IgG} 2$ and $\mathrm{IgG} 3$.

IgG4 deserves a special comment. At present there is a novel disease category, associated with IgG4. They may involve different organs and systems, being characterized by increased IgG4 in serum, a dense lymphoplasmacytic infiltrate rich in IgG4-positive plasma cells, tumefactive lesions with storiform fibrosis, which quickly respond to glucocorticoids [40]. This group includes diseases such as Mikulicz's syndrome, retroperitoneal fibrosis, Küttner's tumor, and Riedel's thyroiditis [41], but not CD.

This review identified only three additional studies, which cannot be compared, assessing antibodies and/or isotypes in celiac patients. Choung et al presented interesting results at the American Gastroenterology Association meeting (AGA 2016) [42]; they evaluated serum 4976 samples from patients that were evaluated for CD. They related the IgG-TTG and IgA-TTG values found and the $\operatorname{IgA}$ status. 5.2\% were completely $\operatorname{IgA}$ deficient, $30.5 \%$ had partial IgA deficiency and $64.3 \%$ showed IgA sufficient values. Distribution of IgG-TTG and IgA-TTG are summarized in Table 1. Presence of HLA risk alleles did not differentiate patients by their IgA status. Whereas it was interesting that among $\operatorname{IgA}$ sufficient patients only $41.2 \%$ of those IgG-TTG positive carried risk alleles for $\mathrm{CD}$.

Table 1. Distribution of IgA and IgG positivity in 4976 serum samples from celiac patients*

\begin{tabular}{|c|c|c|c|}
\hline $\operatorname{IgA}$ & At least one tTG $(+)$ & \multicolumn{2}{|c|}{ Isotypes (in \%) present in positive samples } \\
\hline Complete deficiency & $13.8 \%$ & $100 \% \mathrm{IgG}$ isotype & \\
\hline Partial deficiency & $4.9 \%$ & $85.3 \% \mathrm{IgG}$ isotype & $30.7 \%$ TTG-IgA isotype \\
\hline Normalcy & $4.2 \%$ & $68.1 \%$ TTG-IgG & $50.4 \%$ TTG-IgA \\
\hline
\end{tabular}

*= Based on Choung RS report (44). 
A second study included measurement of IgG-AGA and IgG-TTG in 126 celiac patients with complete IgA deficiency at the time of study [43]. Both antibodies were negative in $6 \%$ of patients. $37 / 40$ who had both antibodies positive accepted a duodenal biopsy and diagnosis was confirmed. In patients with only IgG-AGA positive small intestinal biopsies were all normal and in 11/18 of patients with only IgA-TTG positive patients the biopsy confirmed CD. In all, CD was diagnosed in $11 / 126$ IgA deficient patients $(8.7 \%)$. Authors conclude that measurement of IgG-TTG is recommended for screening of CD in IgA- deficient persons an also, that IgG-AGA should not be used during the routine CD diagnostic process [43]. This is in agreement with Villalta et al [44], who recommended measuring IgG-DGP in these uncertain patients. It seems indeed reasonable that when antibodies are not clear a small intestinal biopsy should continue being the gold standard for diagnosing CD.

Finally, there is a recent study that evaluated 178 patients that proved IgA-TTG negative and IgG-TTG positive during the diagnostic study [45]. Results were contrasted against histological findings; 1/178 demonstrated to have $\mathrm{CD}$, which means that the test was useful in $3 \%$ of patients. In the group assessed, $18 \%$ suffered other autoimmune disorders. A total of 3 patients were diagnosed $\mathrm{CD}$, but none was $\operatorname{Ig} \mathrm{A}$ deficient; of those patients with partial IgA deficiency $(n=72)$ none was celiac. Authors conclude that in this series IgG-TTG did not show association with histological findings and did not help predicting $\mathrm{CD}$, even in those patients with partial selective IgA deficiency.

In summary, the evidence reviewed indicate that: i) measuring IgA-TTG is an effective and helpful tool during the diagnostic process of $\mathrm{CD}$; ii) measuring IgG-TTG shows high sensitivity and specificity in IgA deficient patients, representing a helpful tool in the diagnosis of $\mathrm{CD}$ in IgA deficient patients; iii) antitransglutaminase autoantibodies reflect the autoimmune processes present and not necessarily CD, representing a potential confusion factor during $\mathrm{CD}$ diagnosis; iv) the lower sensitivity and specificity of IgG-TTG makes it not suitable for the routine CD diagnostic process in IgA sufficient individuals.

\section{References}

[1] Husby S, Koletzko S, Korponay-Szabo IR, et al. European Society for Pediatric Gastroenterology, Hepatology, and Nutrition guidelines for the diagnosis of coeliac disease. Journal of pediatric gastroenterology and nutrition. Jan 2012;54(1):136-160.

[2] Hill ID, Fasano A, Guandalini S, et al. NASPGHAN Clinical Report on the Diagnosis and Treatment of Gluten-related Disorders. Journal of pediatric gastroenterology and nutrition. Jul 2016;63(1):156-165.

[3] Catassi C, Kryszak D, Bhatti B, et al. Natural history of celiac disease autoimmunity in a USA cohort followed since 1974. Annals of medicine. Oct 2010;42(7):530-538.

[4] Samasca G, Lerner A, Girbovan AM, et al. Challenging in Glutenfree diet in celiac disease: Prague consensus. European journal of clinical investigation. Mar 302017.

[5] Comino I, Real A, Vivas S, et al. Monitoring of gluten-free diet compliance in celiac patients by assessment of gliadin 33-mer equivalent epitopes in feces. The American journal of clinical nutrition. Mar 2012;95(3):670-677.
[6] Moreno ML, Cebolla A, Munoz-Suano A, et al. Detection of gluten immunogenic peptides in the urine of patients with coeliac disease reveals transgressions in the gluten-free diet and incomplete mucosal healing. Gut. Feb 2017;66(2):250-257.

[7] Lind MV, Madsen ML, Rumessen JJ, et al. Plasma Alkylresorcinols Reflect Gluten Intake and Distinguish between Gluten-Rich and Gluten-Poor Diets in a Population at Risk of Metabolic Syndrome. The Journal of nutrition. Oct 2016;146(10):1991-1998.

[8] Korponay-Szabo IR, Dahlbom I, Laurila K, et al. Elevation of IgG antibodies against tissue transglutaminase as a diagnostic tool for coeliac disease in selective IgA deficiency. Gut. Nov 2003; 52(11) 1567-1571.

[9] Griffin M, Casadio R, Bergamini CM. Transglutaminases: nature's biological glues. The Biochemical journal. Dec 01 2002;368(Pt 2): 377-396.

[10] Wang Z, Griffin M. TG2, a novel extracellular protein with multiple functions. Amino Acids. Feb 2012;42(2-3):939-949.

[11] Rostom A, Dube C, Cranney A, et al. The diagnostic accuracy of serologic tests for celiac disease: A systematic review. Gastroenterology. Apr 2005;128(4):S38-S46.

[12] Brandtzaeg P. The changing immunological paradigm in coeliac disease. Immunology letters. Jun 15 2006;105(2):127-139.

[13] Comerford R, Byrne G, Feighery C, Kelly J. Binding of Autoantibodies to the Core Region of Tissue Transglutaminase Is a Feature of Paediatric Coeliac Disease. Journal of pediatric gastroenterology and nutrition. Oct 2012;55(4):445-450.

[14] Byrne G, Ryan F, Jackson J, Feighery C, Kelly J. Mutagenesis of the catalytic triad of tissue transglutaminase abrogates coeliac disease serum IgA autoantibody binding. Gut. Mar 2007; 56(3): 336-341.

[15] Comerford R, Kelly J, Feighery C, Byrne G. IgG anti-tTG responses in different autoimmune conditions differ in their epitope targets and subclass usage. Molecular immunology. Oct 2015; 67(2 Pt B):369-376.

[16] Konopka E, Grzywnowicz M, Oralewska B, Cielecka-Kuszyk J, Trojanowska I, Cukrowska B. Clinical utility of quantitative multi-antibody Polycheck immunoassays in the diagnosis of coeliac disease. World journal of gastrointestinal pharmacology and therapeutics. May 06 2016;7(2):254-260.

[17] Carroccio A, Vitale G, Di Prima L, et al. Comparison of antitransglutaminase ELISAs and an anti-endomysial antibody assay in the diagnosis of celiac disease: a prospective study. Clinical chemistry. Sep 2002;48(9):1546-1550.

[18] Kaukinen K, Turjanmaa K, Maki M, et al. Intolerance to cereals is not specific for coeliac disease. Scandinavian journal of gastroenterology. Sep 2000;35(9):942-946.

[19] Hansson T, Dahlbom I, Hall J, et al. Antibody reactivity against human and guinea pig tissue transglutaminase in children with celiac disease. Journal of pediatric gastroenterology and nutrition. Apr 2000;30(4):379-384.

[20] Wolters V, Vooijs-Moulaert AF, Burger H, et al. Human tissue transglutaminase enzyme linked immunosorbent assay outperforms both the guinea pig based tissue transglutaminase assay and anti-endomysium antibodies when screening for coeliac disease. European journal of pediatrics. May 2002;161(5):284-287.

[21] Crabbe PA, Heremans JF. Selective IgA deficiency with steatorrhea. A new syndrome. The American journal of medicine. Feb 1967;42(2):319-326.

[22] Kokkonen J, Simila S, Vuolukka P. The incidence of coeliac disease and pyloric stenosis in children in Northern Finland. Annals of clinical research. Jun 1982;14(3):123-128.

[23] Holding S, Wilson F, Spradbery D. Clinical evaluation of the BioPlex 2200 Celiac IgA and IgG Kits - a novel multiplex screen incorporating an integral check for IgA deficiency. Journal of immunological methods. Mar 2014;405:29-34.

[24] Chow MA, Lebwohl B, Reilly NR, Green PH. Immunoglobulin A deficiency in celiac disease. Journal of clinical gastroenterology. Nov-Dec 2012;46(10):850-854.

[25] Sblattero D, Berti I, Trevisiol C, et al. Human recombinant tissue transglutaminase ELISA: an innovative diagnostic assay for celiac disease. The American journal of gastroenterology. May 2000;95(5):1253-1257.

[26] van der Windt DA, Jellema P, Mulder CJ, Kneepkens CM, van der Horst HE. Diagnostic testing for celiac disease among patients 
with abdominal symptoms: a systematic review. Jama. May 05 2010;303(17):1738-1746.

[27] Kim SY, Jeitner TM, Steinert PM. Transglutaminases in disease. Neurochemistry international. Jan 2002;40(1):85-103.

[28] Pereda I, Bartolome-Pacheco MJ, Martin M, Lopez-Escribano H, Echevarria S, Lopez-Hoyos M. Antitissue transglutaminase antibodies in HIV infection and effect of highly active antiretroviral therapy. Journal of acquired immune deficiency syndromes. Aug 15 2001;27(5):507-508.

[29] Peracchi M, Trovato C, Longhi M, et al. Tissue transglutaminase antibodies in patients with end-stage heart failure. The American journal of gastroenterology. Nov 2002;97(11):2850-2854.

[30] Lampasona V, Bonfanti R, Bazzigaluppi E, et al. Antibodies to tissue transglutaminase $\mathrm{C}$ in type I diabetes. Diabetologia. Oct 1999;42(10):1195-1198.

[31] Farrace MG, Picarelli A, Di Tola $M$, et al. Presence of anti-"tissue" transglutaminase antibodies in inflammatory intestinal diseases: an apoptosis-associated event? Cell death and differentiation. Jul 2001;8(7):767-770.

[32] Bizzaro N, Villalta D, Tonutti E, et al. IgA and IgG tissue transglutaminase antibody prevalence and clinical significance in connective tissue diseases, inflammatory bowel disease, and primary biliary cirrhosis. Digestive diseases and sciences. Dec 2003;48(12):2360-2365.

[33] Picarelli A, Di Tola M, Sabbatella L, et al. Anti-tissue transglutaminase antibodies in arthritic patients: a disease-specific finding? Clinical chemistry. Dec 2003;49(12):2091-2094.

[34] Roth EB, Stenberg P, Book C, Sjoberg K. Antibodies against transglutaminases, peptidylarginine deiminase and citrulline in rheumatoid arthritis--new pathways to epitope spreading. Clinical and experimental rheumatology. Jan-Feb 2006;24(1):12-18.

[35] Villalta D, Bizzaro N, Tonutti E, Tozzoli R. IgG antitransglutaminase autoantibodies in systemic lupus erythematosus and Sjogren syndrome. Clinical chemistry. Jul 2002;48(7):1133.

[36] Sardy M, Csikos M, Geisen C, et al. Tissue transglutaminase ELISA positivity in autoimmune disease independent of glutensensitive disease. Clinica chimica acta; international journal of clinical chemistry. Feb 2007;376(1-2):126-135.

[37] Riente L, Chimenti D, Pratesi F, et al. Antibodies to tissue transglutaminase and Saccharomyces cerevisiae in ankylosing spondylitis and psoriatic arthritis. The Journal of rheumatology. May 2004;31(5):920-924.

[38] Cataldo F, Lio D, Marino V, Picarelli A, Ventura A, Corazza GR $\mathrm{IgG}(1)$ antiendomysium and $\mathrm{IgG}$ antitissue transglutaminase (antitTG) antibodies in coeliac patients with selective IgA deficiency. Working Groups on Celiac Disease of SIGEP and Club del Tenue. Gut. Sep 2000;47(3):366-369.

[39] Agardh D, Borulf S, Lernmark A, Ivarsson SA. Tissue transglutaminase immunoglobulin isotypes in children with untreated and treated celiac disease. Journal of pediatric gastroenterology and nutrition. Jan 2003;36(1):77-82.

[40] Stone JH, Khosroshahi A, Deshpande V, et al. Recommendations for the nomenclature of IgG4-related disease and its individual organ system manifestations. Arthritis and rheumatism. Oct 2012;64(10):3061-3067

[41] Dahlgren M, Khosroshahi A, Nielsen GP, Deshpande V, Stone JH. Riedel's thyroiditis and multifocal fibrosclerosis are part of the IgG4-related systemic disease spectrum. Arthritis Care Res (Hoboken). Sep 2010;62(9):1312-1318.

[42] Choung RS JRM, Manish J. Gandhi, Joseph A. Murray, Melissa Snyder Role of Immunoglobulin A and Tissue Transglutaminase IgG in Celiac Disease Evaluation. AGA conference 2016. Vol 2016:S314 Sa1433.

[43] Lenhardt A, Plebani A, Marchetti F, et al. Role of human-tissue transglutaminase IgG and anti-gliadin IgG antibodies in the diagnosis of coeliac disease in patients with selective immunoglobulin A deficiency. Digestive and liver disease. official journal of the Italian Society of Gastroenterology and the Italian Association for the Study of the Liver. Nov 2004; 36(11): 730-734.

[44] Villalta D, Alessio MG, Tampoia M, et al. Testing for IgG class antibodies in celiac disease patients with selective IgA deficiency. A comparison of the diagnostic accuracy of 9 IgG anti-tissue transglutaminase, $1 \mathrm{IgG}$ anti-gliadin and $1 \mathrm{IgG}$ anti-deaminated gliadin peptide antibody assays. Clinica chimica acta: international journal of clinical chemistry. Jul 2007;382(1-2): 95-99.

[45] Absah I, Rishi AR, Gebrail R, Snyder MR, Murray JA. The Lack of Utility of Anti-tTG IgG in the Diagnosis of Celiac Disease When Anti-tTG IgA Is Negative. Journal of pediatric gastroenterology and nutrition. Sep 122016. 